\title{
Vacunación contra el virus del papiloma humano (HPV) para prevenir el cáncer de cuello uterino y sus precursores: una evidencia válida a pesar de las críticas
}

Vaccination against human papillomaviruses (HPV) to prevent cervical cancer and its precursors: a valid evidence despite criticism

Arbyn M y col. Cochrane Database of Systematic Reviews 2018, Issue 5. Art. No.: CD009069

\section{Objetivos}

Evaluar la protección contra las lesiones precancerosas/cancerosas y la infección por el virus del papiloma humano (HPV) 1618 en mujeres adolescentes y adultas.

\section{Fuentes de datos}

Búsqueda en MEDLINE, Cochrane y Embase de artículos publicados hasta Junio de 2017. También fueron buscados estudios no publicados sobre efectos adversos y mortalidad en registros de ensayos clínicos y en los registros de los fabricantes de la vacuna.

\section{Selección de estudios}

Estudios controlados y aleatorizados que hubieran comparado con placebo la seguridad y la eficacia de la vacuna contra el HPV.

\section{Extracción de los datos y análisis}

Se utilizó GRADE para valorar la calidad de la evidencia sobre la protección contra lesiones intraepiteliales cervicales (CIN) 2, CIN 3 y carcinoma in situ, y sobre eventos adversos. Los resultados fueron lesiones precancerosas/cancerosas asociadas con los serotipos de HPV de las vacunas y los que no estaban en las vacunas. Los resultados se presentan en forma de riesgo relativo (RR) y reducción del riesgo relativo (RRR).

\section{Resultados principales}

Considerando el efecto de la vacuna sobre la incidencia de CIN2, independientemente del tipo del estado de HPV basal de la mujer (el más importante epidemiológicamente), la RRR de desarrollar este tipo de lesiones fue del 30\% (IC95\% de 15 a $42 \%$ ) entre las de 15 a 26 años, pero no se apreciaron efectos importantes en el grupo de 24 a 45 años: RRR 4\% (-30 a +17\%). Independientemente del tipo del estado de HPV, las mujeres de 15 a 26 años presentaron una RRR de desarrollar lesiones CIN3 de $33 \%$ (IC95\% de 7 a $51 \%$ ) y de desarrollar carcinoma in situ de $68 \%$ (33 a $85 \%$ ). No se encontraron datos discriminados entre CIN3 y carcinoma in situ para el grupo de mayor edad. La certeza de la evidencia y el impacto en valores absolutos puede apreciarse en la tabla 1 que resume los principales efectos de seguridad y eficacia. No se observó que la vacuna aumente la incidencia de abortos, terminaciones del embarazo o de otros efectos adversos serios. Podría aumentar las muertes maternas, las anomalías congénitas y los mortinatos, sin embargo, el amplio intervalo de confianza también incluye un efecto protector.

Tabla 1. Efectos de la vacuna contra el virus del papiloma humano sobre la incidencia de lesiones cervicales, eventos adversos y muerte en niñas, adolescentes y mujeres no seleccionadas para el estado del ADN del HPV al inicio del estudio.

\begin{tabular}{|c|c|c|c|c|c|c|c|}
\hline \multirow{2}{*}{ Resultados } & \multirow[t]{2}{*}{$\begin{array}{c}\text { Edad en } \\
\text { años }\end{array}$} & \multirow[t]{2}{*}{ Seguimiento } & \multicolumn{2}{|c|}{$\begin{array}{l}\text { Efectos absolutos } \\
\text { anticipados (IC 95\%) }\end{array}$} & \multirow[t]{2}{*}{$\begin{array}{l}\text { Riesgo relativo } \\
\text { (IC 95\%) }\end{array}$} & \multirow{2}{*}{$\begin{array}{l}\text { Número de } \\
\text { participantes } \\
\text { (estudios) }\end{array}$} & \multirow{2}{*}{$\begin{array}{l}\text { Certeza de la } \\
\text { evidencia } \\
\text { (GRADE) }\end{array}$} \\
\hline & & & Placebo & Vacuna & & & \\
\hline \multirow[t]{2}{*}{ CIN2+a } & 15 a 26 & $\begin{array}{c}3,5 \text { a } \\
\text { 8,5 años }\end{array}$ & $559 / 10.000$ & $\begin{array}{l}391 / 10.000 \\
(324 \text { a } 475)\end{array}$ & $\begin{array}{c}0,70 \\
(0,58 \text { a } 0,85) \\
\end{array}$ & $\begin{array}{l}35.779 \\
(4 \mathrm{ECA})\end{array}$ & $\begin{array}{c}\oplus \oplus \oplus \oplus \\
\text { ALTA }\end{array}$ \\
\hline & 24 a 45 & $\begin{array}{c}3,5 \text { a } \\
6 \text { años }\end{array}$ & $343 / 10.000$ & $\begin{array}{l}356 / 10.000 \\
(284 \text { a } 445)\end{array}$ & $\begin{array}{c}1,04 \\
(0,83 \text { a } 1,30)\end{array}$ & $\begin{array}{c}9287 \\
(2 \mathrm{ECA})\end{array}$ & $\begin{array}{c}\oplus \oplus \oplus \ominus \\
\text { MODERADA }\end{array}$ \\
\hline CIN3+a & 15 a 26 & $\begin{array}{c}3,5 \text { a } \\
4 \text { años }\end{array}$ & $266 / 10.000$ & $\begin{array}{l}178 / 10.000 \\
\text { (231 a } 247) \\
\end{array}$ & $\begin{array}{c}0,67 \\
(0,49 \text { a } 0,93) \\
\end{array}$ & $\begin{array}{l}35.489 \\
(3 \mathrm{ECA}) \\
\end{array}$ & $\begin{array}{c}\oplus \oplus \oplus \ominus \\
\text { MODERADA }\end{array}$ \\
\hline \multicolumn{2}{|c|}{$\begin{array}{l}\text { Adenocarcinoma in situ asociado } \\
\text { a HPV } 16 \text { ó } 18\end{array}$} & 3,5 años & $14 / 10.000$ & $\begin{array}{c}5 / 10.000 \\
(3 \text { a } 11)\end{array}$ & $\begin{array}{c}0,36 \\
(0,17 \text { a } 0,78)\end{array}$ & $\begin{array}{l}34.562 \\
(2 \mathrm{ECA})\end{array}$ & $\begin{array}{c}\oplus \oplus \oplus \oplus \\
\text { ALTA }\end{array}$ \\
\hline \multicolumn{2}{|c|}{ Eventos adversos serios } & $\begin{array}{l}6 \text { meses a } \\
7 \text { años } \\
\end{array}$ & $669 / 10.000$ & $\begin{array}{l}656 / 10.000 \\
(616 \text { a } 703) \\
\end{array}$ & $\begin{array}{c}0,98 \\
(0,92 \text { a } 1,05) \\
\end{array}$ & $\begin{array}{l}71.597 \\
(23 \mathrm{ECA}) \\
\end{array}$ & $\begin{array}{l}\oplus \oplus \oplus \oplus \\
\text { ALTA }\end{array}$ \\
\hline \multicolumn{2}{|l|}{ Muertes } & $\begin{array}{c}7 \text { meses a } \\
10 \text { años }\end{array}$ & $11 / 10.000$ & $\begin{array}{c}14 / 10.000 \\
(9 \text { a } 22)\end{array}$ & $\begin{array}{c}1,29 \\
(0,85 \text { a } 1,98)\end{array}$ & $\begin{array}{c}71.176 \\
(23 \text { ECA) }\end{array}$ & $\begin{array}{c}\oplus \oplus \Theta \Theta \\
\text { BAJA }\end{array}$ \\
\hline
\end{tabular}

a: Cualquier tipo de HPV. IC: intenvalo de confianza. CIN: neoplasia intraepitelial cenvical.

\section{Conclusión}

La vacuna protege contra lesiones premalignas en mujeres de 15 a 26 años y probablemente en mujeres mayores. No tendría un efecto importante en cuanto a efectos adversos serios.

Fuentes de financiamiento: Scientific Institute of Public Health (Brussels),
Belgium. National Institute for Health Research, UK. Otras instituciones no gubernamentales y gubernamentales. Conflicto de interés: tres autores principales recibieron becas de viaje de MSD-Sanofi-Pasteur y GSK hasta 2008. Esto fue evaluado por el órgano de arbitraje de Cochrane quien autorizó su autoría bajo las estrictas políticas de conflictos de intereses.

\section{Comentario}

El cáncer de cuello uterino es un problema que causa un gran número de muertes en mujeres a nivel mundial, especialmente entre las poblaciones más vulnerables y con menor acceso a los programas de cribado. Se estima que es la cuarta causa de muerte por cáncer a nivel mundial y las estrategias para prevenirlo son de interés global ${ }^{1}$. Medio millón de mujeres son diagnosticadas con cáncer de cuello uterino anualmente ( $85 \%$ provienen de países de bajos y medianos ingresos), y la mitad de estas mujeres mueren a causa de su enfermedad. Existe una eficaz estrategia de prevención secundaria como es el rastreo con Papanicolaou.
En los últimos años se ha agregado una estrategia de prevención primaria a través de la vacuna contra el HPV (principal agente etiológico de dicho cáncer), actualmente recomendada por la Organización Mundial de la Salud (OMS) y adoptada a la fecha por 84 países de diferentes continentes y niveles de ingresos². La revisión Cochrane evaluó la seguridad y efectividad de dicha vacuna. Si bien no encontró evidencia directa sobre su efecto sobre la incidencia de cáncer cervical, identificó evidencia de moderada a alta certeza de su efecto preventivo sobre lesiones premalignas de cáncer de cuello uterino, consideradas por la OMS como de- 
senlaces subrogantes o intermedios válidos, dado el prolongado tiempo de seguimiento y la magnitud del tamaño muestral que se necesitaría para valorar como desenlace al cáncer cervical ${ }^{3}$. Si bien el efecto sobre lesiones premalignas asociadas al HPV 16/18 fue clínica y estadísticamente significativo, cuando se considera el efecto sobre las lesiones, independientemente del status basal HPV de la mujer (el resultado más importante desde el punto de la validez externa), solo pudo documentarse un efecto en el grupo etario más joven, que por otra parte es el que debería ser destinatario de las mayores acciones preventivas. Aunque este metaanálisis documentó con moderada a alta certeza la evidencia de la eficacia de la vacuna para prevenir lesiones precursoras de cáncer de cuello uterino, para inferir que podría reducir el cáncer cervical, esta certeza merece degradarse al menos un nivel por la aplicación de evidencia indirecta (a baja o moderada certeza de la evidencia). No hubo efectos adversos serios, sin embargo los ECA nunca tienen el tamaño ni el seguimiento necesarios para evaluar todos los efectos adversos infrecuentes que la farmacovigilancia sí puede identificar. Por otro lado y como era de esperarse, la evidencia sobre la mortalidad es inconsistente e imprecisa. El 27 de julio de 2018, se publicó un artículo en la revista British Medical Journal Evidence Based Medicine (BMJ-EBM) que criticaba la reciente Revisión Cochrane sobre las vacunas profilácticas del virus del papiloma humano (HPV) ${ }^{4}$. Este artículo se basó en análisis realizados en el Centro Cochrane Nórdico y fue avalado por dos experimentados investigadores Cochrane: los profesores Peter Gøtzsche y Tom Jefferson. La más notable de las críticas que hicieron estos investigadores fue que la Revisión Cochrane estaba incompleta debido a la omisión de "casi la mitad de los ensayos elegibles". Tomando muy en serio todas las críticas y comentarios, Cochrane inició una investigación, trabajando con los autores y los editores de la revisión y con investigadores independientes, que no habían participado en la publicación original. Posteriormente, el editor en jefe publicó una respuesta a cada una de las críticas ${ }^{5}$. La Revisión Cochrane cuestionada sólo había perdido un pequeño número de estudios debido a que su estrategia principal había estado basada en identificar estudios publicados en revistas científicas revisados por pares, lo que ya había sido declarado en el protocolo revisado por pares y publicado en diciembre de 2013. Sin embargo, la lista de otros 20 estudios "potencialmente elegibles" basada en los criterios de inclusión de la Revisión Cochrane, llamativamente no se incluyó como apéndice del artículo ni fue provista tras ser solicitada a los autores. La revisión Cochrane criticada había incluido 26 ensayos (73.428 participantes) que habían cumplido con los criterios de inclusión predeterminados. La búsqueda ampliada identificó además otro cinco estudios elegibles (con un total de 5267 mujeres) que habían sido omitidos por no haber buscado en registros de ensayos clínicos, lo que, si bien no es un problema menor, dista mucho de los 20 estudios (48.276 mujeres) que afirmaban Jørgensen y col. que habían sido omitidos. Además, luego del re-análisis pudo constatarse que el agregado de los estudios no publicados tuvo escasa o nula influencia en los resultados principales. La nueva versión actualizada está avanzando rápidamente y en breve se publicará incluyendo todos los datos relevantes, públicamente disponibles. El artículo BMJ-EBM también planteó algunas preocupaciones sobre los comparadores utilizados en los ensayos, que se basaron en aluminio, como se describe claramente en el resumen, en la sección de métodos y también en la tabla de características detalladas de estudios de la revisión. Es decir, los comparadores de ensayos se describieron de forma inequívoca, transparente y precisa. Algunos grupos argumentan que los controles en los estudios deberían haber recibido agua o solución salina para prevenir enmascaramiento de los daños causados por la administración de sales de aluminio a ambos grupos en los estudios. Sin embargo, los resultados de eficacia en nada se verían afectados por el uso en ambos grupos de este coadyuvante tan común. Por otro lado, uno de los autores del artículo de BMJ-EBM (Tom Jefferson) publicó una revisión sistemática en 2004 que no encontró evidencia de daños graves o a largo plazo de las sales de aluminio y concluyó que no se justificaba investigación adicional ${ }^{6}$. Igualmente una nueva Revisión Cochrane en marcha examinará la seguridad del aluminio en todas las vacunas ${ }^{7}$. También fue cuestionado el uso de resultados intermedios ("subrogantes") en consonancia con las recomendaciones de la OMS. Si bien la progresión de CIN2 y CIN3 a cáncer no es inexorable sin tratamiento, de todas formas, claramente aumenta el riesgo en presencia de este tipo de lesiones precursoras, razón por la cual ambas condiciones están sujetas a tratamiento. El riesgo de progresión a cáncer aumenta a medida que progresan las lesiones, lo que se previene de manera efectiva tratándolas. No sería ético que los investigadores de un eventual ECA cuyo desenlace final sea el cáncer cervical dejen sin tratar los estados precursores, por lo sería esperable la ausencia casi completa de cáncer de cuello uterino en cualquier brazo de dicho ensayo. Por lo tanto, además de antiético, dicho ECA tampoco sería factible. Probablemente debamos esperar muchos años y una serie de tiempo interrumpida (poderoso diseño cuasiexperimental) proveniente de los países que implementaron la vacunación, e idealmente usando como grupos control a los que no la implementaron, para obtener una evidencia definitiva sobre el efecto sobre el cáncer cervical. Asimismo, este tipo de diseño permitirá explorar el efecto secular de la vacunación y el desconocido impacto sobre la ecología de otros serotipos oncogénicos no cubiertos por la vacuna ${ }^{8,9}$. Se planteó también que la Revisión Cochrane revisó incompletamente los eventos adversos serios y citaron como ejemplo el estudio PATRICIA. Según los informes de ClinicalTrials.gov y del registro de estudios de GlaxoSmithKline (GSK), las cifras concuerdan exactamente. Con respecto al exceso de muertes en las mujeres mayores vacunadas, los datos se presentaron de forma transparente, pero también para proporcionar un contexto más completo, se incluyó la evaluación de los expertos de la OMS que no encontraron un claro mecanismo causal de la vacuna. El informe de otros daños fue, como se describe en el protocolo, limitado a los ECA publicados en revistas revisadas por pares. En la sección de la discusión de la Revisión Cochrane se destacó la importancia de los programas nacionales de vigilancia para identificar y reportar daños infrecuentes o de largo plazo. Esto es particularmente cierto cuando se trata de daños tales como síndromes de disfunción autonómica y otros síndromes que han generado preocupación a partir de reportes observacionales. Se cuestionó también la presencia de conflicto de intereses, sin embargo, la revisión cumplió con la estricta política Cochrane de conflicto de intereses. Esta colaboración considera clave a la declaración, pero reconoce que puede ser insuficiente. Por eso, en circunstancias específicas, algunos investigadores son excluidos de la participación en una revisión como parte de un equipo de autores. En relación con la revisión de vacunas contra el HPV, Cochrane recibió comentarios luego de la publicación del protocolo. El primer autor había invitado a un equipo de investigadores de vacunación contra el HPV con el propósito de ayudar a obtener datos no publicados. Todos estos expertos habían declarado sus conflictos, pero su inclusión hizo que el equipo inicialmente propuesto por el autor no cumpliera con la política de Cochrane. Por lo tanto, se hicieron cambios que aseguraron que el trabajo de la revisión fuera realizado por un equipo cuyos miembros activamente involucrados en la realización de la revisión cumplieran completamente con los lineamientos de dicha política. Jørgensen y col. también declararon que el principal autor de la revisión lidera la vigilancia post-comercialización de la Agencia de Medicina Europea (EMA) y la vinculó a la financiación de un fabricante. En realidad, el estudio de vigilancia no fue financiado por la EMA ni por ningún fabricante de vacunas. También denunciaron que el 
ensayo de Costa Rica no había sido financiado con fondos públicos, como reportaba la revisión, sino por GSK. Este no fue el caso, como se señaló en la declaración de conflicto de intereses en el informe publicado del estudio en $\mathrm{JAMA}^{10}$ que indicó que fue "... financiado por el $\mathrm{NCl}$ (subvención N01-CP-11005), con fondos de apoyo de la Oficina de Institutos Nacionales de Salud para la Salud de la Mujer y del Ministerio de Salud de Costa Rica. La vacuna fue provista por GSK Biologicals, en virtud de un acuerdo de ensayos clínicos con el $\mathrm{NCl}$...".

Contrariamente a la crítica, la cobertura mediática Cochrane fue cautelosa y equilibrada, pero la Unidad Editorial Cochrane reconoce que podría haber mejoras en relación con la transparencia cuando se citan expertos externos.

En resumen, el artículo publicado en BMJ-EBM exageró sustancialmente sus críticas. Algunas de ellas constituyen aportes valiosos que se reflejarán en la actualización en curso. Es saludable y necesario el reanálisis independiente de cualquier investigación, tal como ocurrió con los conocidos caso del "Tamiflugate"11 o de la Paroxetina ${ }^{12}$. Sin embargo, tales aportes se opacan cuando se les imprime un tono sensacionalista que lo aleja del ámbito científico. Ya existe un formidable y creciente lobby antivacunación. El resultado de esta controversia en un marco inapropiado no sería inocuo, pues una menor cobertura de la vacuna podría provocar que muchas mujeres sufran y mueran innecesariamente por cáncer de cuello uterino.

\section{Conclusiones de los comentadores}

La vacunación contra el HPV constituye una sólida y válida evidencia con respecto a la prevención de lesiones precursoras, aunque no definitiva en cuanto al cáncer cervical. Por otro lado, debemos destacar que es necesario mantener un pensamiento crítico a la hora de valorar nuevos hallazgos científicos, independientemente de lo prestigiosa que pueda ser la fuente de la evidencia. Un juicioso uso de las herramientas de lectura crítica que brinda la Medicina Basada en la Evidencia y un cuidadoso análisis del balance riesgo-beneficio nos permitirán sacar mejores conclusiones y limitar el riesgo de sesgo entre los que solapadamente pueden filtrarse también los conflictos de intereses.

Agustín Ciapponi [ Servicio de Medicina Familiar y Comunitaria del Hospital Italiano de Buenos Aires. Director de Cochrane Argentina y del Centro Cochrane IECS ]

Nahuel Orcaizaguirre [ Servicio de Medicina Familiar y Comunitaria del Hospital Italiano de Buenos Aires. nahuel.orcaizaguirre@ hospitalitaliano.org.ar ]

Ciapponi A, Orcaizaguirre N. Vacunación contra el virus del papiloma humano (HPV) para prevenir el cáncer de cuello uterino y sus precursores: una evidencia válida a pesar de las críticas. Evid Actual Pract Ambul 2018;21(3):80-82. Comentado de: Arbyn M y col. Prophylactic vaccination against human papillomaviruses to prevent cervical cancer and its precursors. Cochrane Database of Systematic Reviews 2018 , Issue 5. Art. No.: CD009069. DOI: 10.1002/14651858.CD009069.pub3

\section{Referencias}

1. Ferlay y col. Cancer incidence and mortality worldwide: sources, methods and major patterns in GLOBOCAN 2012. International journal of cancer, 2015, vol. 136, no 5, p. E359-E386.

2. WHO vaccine-preventable diseases: monitoring system. 2018 global summary. Available at http://www.who.int/entity/immunization/monitoring_surveillance/data/year_vaccine_introduction.xls?ua=1. Accesed 2018/October/6

3. Pagliusi S y col. Efficacy and other milestones for human papillomavirus vaccine introduction. Vaccine, 2004, vol. 23, no 5, p. 569-578.

4. Jørgensen L y col. The Cochrane HPV vaccine review was incomplete and ignored important evidence of bias. BMJ evidence-based medicine, 2018 , p. bmjebm-2018-111012. 5. David Tovey D y col. Cochrane's Editor in Chief responds to BMJ EBM article criticizing HPV review: Cochrane; 2018 [cited 2018 3/9/2018]. Available from: https://www. cochrane.org/news/cochranes-editor-chief-responds-bmj-ebm-article-criticizing-hpv-review.

6 . Jefferson T y col. Adverse events after immunisation with aluminium-containing DTP vaccines: systematic review of the evidence. Lancet Infectious Diseases 2004:4(2):84-90. http://doi.org/10.1016/S1473-3099(04)00927-2

7. Djurisic S y col. Aluminium adjuvants used in vaccines versus placebo or no intervention. Cochrane Database of Systematic Reviews 2017;(9):CD012805. https://doi. org/10.1002/14651858.CD012805

8. Murall C y col. Revising ecological assumptions about Human papillomavirus interactions and type replacement. J Theor Biol. 2014 Jun 7;350:98-109. doi: 10.1016/j. jtbi.2013.12.028. Available from https://www.ncbi.nlm.nih.gov/pubmed/24412334

9. Pons-Salort M y col. Exploring individual HPV coinfections is essential to predict HPV-vaccination impact on genotype distribution: a model-based approach. Vaccine. 2013 Feb 6;31(8):1238-45. doi: 10.1016/j.vaccine.2012.11.098. Available from https://www.ncbi.nlm.nih.gov/pubmed/23246257

10. Hildesheim A y col. Effect of human papillomavirus 16/18 L1 viruslike particle vaccine among young women with preexisting infection: a randomized trial. JAMA 2007:298(7):74353. https://doi.org/10.1001/jama.298.7.743

11. Ciapponi A. Del escándalo del Tamiflu a una revolución de la evidencia científica en salud. Evid Act Pract Ambul Abril-Junio 2014; 17(2):42-45. http://www.evidencia.org.ar/ hitalba-pagina-articulo.php?cod_producto $=4221$ \&origen $=2$

12. Ciapponi A. La cara oculta del estudio 329 y la manipulación de la evidencia científica. Evid Act Pract Ambul. 2016;19(3):71-75. https://www.fundacionmf.org.ar/visor-producto. php?cod_producto $=4788$ 